\title{
Perspectives, perceptions and experiences in postoperative pain management in developing countries: A focus group study conducted in Rwanda
}

\author{
Ana P Johnson PhD ${ }^{1}$, Ryan Mahaffey MD FRCPC ${ }^{2}$, Rylan Egan $\mathrm{PhD}^{3}$, Theogene Twagirumugabe MD ${ }^{4}$, Joel L Parlow MD FRCPC ${ }^{2}$
}

AP Johnson, R Mahaffey, R Egan, T Twagirumugabe, JL Parlow. Perspectives, perceptions and experiences in postoperative pain management in developing countries: A focus group study conducted in Rwanda. Pain Res Manag 2015;20(5):255-260.

BACKGROUND: Access to postoperative acute pain treatment is an important component of perioperative care and is frequently managed by a multidisciplinary team of anesthesiologists, surgeons, pharmacists, technicians and nurses. In some developing countries, treatment modalities are often not performed due to scarce health care resources, knowledge deficiencies and cultural attitudes.

OBJECTIVES: In advance of a comprehensive knowledge translation initiative, the present study aimed to determine the perspectives, perceptions and experiences of anesthesia residents regarding postoperative pain management strategies.

METHODS: The present study was conducted using a qualitative assessment strategy in a large teaching hospital in Rwanda. During two sessions separated by seven days, a 10-participant semistructured focus group needs analysis was conducted with anesthesia residents at the Centre Hospitalier Universitaire de Kigali (Kigali, Rwanda). Field notes were analyzed using interpretative and descriptive phenomenological approaches. Participants were questioned regarding their perspectives, perceptions and experiences in pain management.

RESULTS: The responses from the focus groups were related to five general areas: general patient and medical practice management; knowledge base regarding postoperative pain management; pain evaluation; institutional/ system issues related to protocol implementation; and perceptions about resource allocation. Within these areas, challenges (eg, communication among stakeholders and with patients) and opportunities (eg, on-the-job training, use of protocols, routine pain assessment, participation in resource allocation decisions) were identified.

CONCLUSIONS: The present study revealed the prevalent challenges residents perceive in implementing postoperative pain management strategies, and offers practical suggestions to overcoming them, primarily through training and the implementation of practice recommendations.

Key Words: Focus group; Perioperative outcomes; Postoperative pain; Rwanda

$\mathrm{R}$ elief from pain is a fundamental human right, as indicated in the 1948 Universal Declaration of Human Rights $(1,2)$ and in the International Association for the Study of Pain Declaration of Montreal (3). The International Association for the Study of Pain, which includes members representing $>200$ countries, has determined the need for health care professionals to increase their knowledge of pain management (3). The WHO, which reports on the global burden of disease, leading causes of death, life expectancy, and adult and child mortality risks, does not provide data regarding quality of health care, measures of suffering or pain (4-9).

\author{
Les perspectives, les perceptions et les expériences \\ en matière de gestion de la douleur postopératoire \\ dans les pays en développement : étude d'un groupe \\ de travail au Rwanda
}

HISTORIQUE : L'accès au traitement de la douleur postopératoire aiguë
est un élément important des soins postopératoires, souvent géré par une
équipe multidisciplinaire d'anesthésistes, de chirurgiens, de pharmaciens,
de techniciens et d'infirmières. Dans certains pays en développement, ces
modalités thérapeutiques sont souvent négligées en raison de la pénurie des
ressources de santé, des lacunes sur le plan des connaissances et des atti-
tudes culturelles. tudes culturelles.

savoir, la présente étude visait à déterminer les perspectives, les perceptions et les expériences des résidents en anesthésie à l'égard des stratégies de gestion de la douleur postopératoire.

MÉTHODOLOGIE : Les chercheurs ont réalisé la présente étude dans un grand hôpital universitaire du Rwanda au moyen d'une stratégie d'évaluation qualitative. Pendant deux séances à sept jours d'intervalle, ils ont effectué une analyse des besoins auprès d'un groupe de travail semistructuré de dix résidents en anesthésie du Centre hospitalier universitaire de Kigali, au Rwanda. Ils ont analysé les notes sur le terrain à l'aide d'approches phénoménologiques interprétatives et descriptives. Ils se sont informés des perspectives, des perceptions et des expériences des participants en matière de gestion de la douleur.

RÉSULTATS : Les réponses des groupes de travail se divisaient en cinq grands secteurs : gestion générale du patient et de l'exercice de la médecine, connaissances sur la gestion de la douleur postopératoire, problèmes de l'établissement ou du système à l'égard de l'adoption de protocoles et perceptions sur l'affectation des ressources. Dans ces secteurs, les chercheurs ont répertorié les problèmes (p. ex., communication entre intervenants et avec les patients) et les possibilités (p. ex., formation en milieu de travail, recours à des protocoles, évaluation systématique de la douleur, participation aux décisions en matière d'affectation des ressources).

CONCLUSIONS : Le présent article révèle les problèmes que les résidents jugent fréquents dans l'adoption des stratégies de gestion de la douleur postopératoire et contient des suggestions pratiques afin de les résoudre, particulièrement par la formation et la mise en œuvre de recommandations sur la pratique.

Severe pain is a characteristic of many medical conditions, and appropriate pain management is at the forefront of perioperative care after major surgery. Inappropriate or inadequate pain management treatments can have several adverse effects on patient well-being, including prolonged immobility and potential complications involving the cardiovascular, respiratory and gastrointestinal systems (10). Furthermore, chronic pain conditions may ensue if acute pain is not appropriately managed $(11,12)$. Adequate pain relief can usually be attained with the use of inexpensive drugs and treatments; however, physicians in developing countries may have limited access to the

${ }^{1}$ Department of Public Health Sciences; ${ }^{2}$ Department of Anesthesiology and Perioperative Medicine; ${ }^{3}$ Centre for Teaching and Learning,

Queen's University, Kingston, Ontario; ${ }^{4}$ Department of Anesthesiology, Critical Care and Emergencies, University of Rwanda, Butare, Rwanda Correspondence: Dr Joel Parlow, Anesthesiology, Kingston General Hospital, 76 Stuart Street, Kingston, Ontario K7L 2V7.

Telephone 613-533-2952, e-mail parlowj@queensu.ca 
pharmaceuticals, technologies and training that would enable them to appropriately manage acute pain. Data from Nigeria (13) indicated that only $50 \%$ of all patients experiencing pain in the emergency department received analgesia and, of these, $80 \%$ still experienced moderate to severe residual pain. Furthermore, a postoperative pain study from Nigeria (14) found that two-thirds of patients continued to experience moderate to unbearable pain $24 \mathrm{~h}$ after surgery.

In developing countries, several aspects of health policy appear to be prioritized without emphasizing pain management. Such areas include increasing access to health care services (especially rural populations), addressing the lack of trained personnel, finances, capital resources, infrastructure and the fragmentation of care (15). Health care is often delivered at clinics (with or without physicians) serving large geographical areas, and the majority of the population living in cities is not able to afford treatment. Treatment of malaria, tuberculosis and HIV/AIDS tends to be given a higher priority than the provision of analgesia. Reliable running water and electricity, which are not universally available, also has to be addressed (16). In Rwanda, cultural perceptions regarding analgesia means that some patients and health care staff have a disproportionate fear of side effects or addiction from its use. These fears may cause patients to suffer from pain in silence, rather than seek treatment (17). Finally, staff members are often overstretched and may not be available to administer analgesic drugs safely, due to lack of time and scarce training.

In a report by the Agency for Health Care Policy and Research, barriers to pain treatment have been classified as system, professional and patient related (18). System barriers occur at the institutional and policy levels and relate to legal and regulatory obstacles based on the potential opioid abuse and addiction, or to resource limitations. Professional barriers occur at the level of the health care workers and can be influenced by physicians treating patients based on their disease or prognosis rather than their pain intensity, lack of education and guideline compliance, fear of patient addiction and poor knowledge of opioid pharmacology $(19,20)$. Patient barriers can encompass attitudinal issues, such as fear of addiction to opioids, an increased tolerance to medication and anxiety about side effects (19-22). Patient barriers may also include fatalism (the belief that suffering is inevitable, predetermined and unavoidable) and psychological elements (anxiety, distress, depression, anger, dementia) (22).

\section{Background to the study}

Through international educational initiatives, such as those of the Canadian Anesthesiologists' Society International Education Foundation (CASIEF) (23), Western medical faculty are increasingly involved in the education of local physicians, residents and allied personnel in developing countries. These initiatives provide tremendous opportunities for improving health outcomes by surveying the existing local knowledge related to values, prioritization of available health care resources and training needs. Any facilitation of knowledge transfer from developed countries to developing countries must consider locally relevant and applicable clinical practices. In addition, education and practice changes must be applied in a culturally and ethically guided manner, which reflect the values and norms of the community, if communication and decision making about the appropriate use of heath care resources is to be improved.

CASIEF has been present in Rwanda since 2006, providing educational assistance in the Anesthesia Residency Training Program at the University of Rwanda, based in Kigali and Butare, Rwanda (previously modelled in Nepal [24] with capacity building and sustainability goals). Clinical training, as well as didactic courses, form the majority of the curriculum. Funds are also provided to enable Rwandan anesthesia staff and residents to attend international meetings and obtain additional training in Western institutions. The emphasis of the program has been on assisting Rwandan physicians in creating their own anesthesia training program, which in turn would positively impact anesthesia safety and available resources (25). The program has been growing steadily since 2006 , and is currently training up to five residents per year $(19,26)$. Thirteen residents have graduated from the program since its inception; many have assumed staff positions in Rwanda, with some pursuing further training abroad.

The following needs analysis was conducted at the Centre Hospitalier Universitaire de Kigali (CHUK), a public university hospital in Kigali, Rwanda. CHUK was built in 1918 and has 509 beds. Its main priorities are patient care, education, research and community service. This university teaching hospital, located in the centre of Kigali (District of Nyarugenge), is the main public health institution in Rwanda, and serves $>1.2$ million individuals from a primarily urban region. CHUK is one of four referral hospitals in Rwanda (the others include the Centre Hospitalier Universitaire de Butare in Butare [Faculty of Medicine; National University of Rwanda, University Laboratory], King Faisal Hospital in Kigali [public-private hospital providing highly specialized services to private patients, patients with private insurance and patients referred from the other referral hospitals] and Rwanda Military Hospital). Patients in Rwanda are assessed through an organized network that includes local health districts, district hospitals and these four referral hospitals. CHUK is unique in that it is the only public general hospital serving a largely urban community located in Kigali. From the point of view of surgical services in Rwanda, the majority of procedures are performed in district hospitals, with CHUK serving as a referral centre for a population of $>6$ million. Surgical volume at CHUK consists of a total of 7682 procedures per annum, with 4164 of these classified as major surgery (2010 data) (27).

At a voluntary educational rotation with the CASIEF program in Rwanda (23) during a three-week period from November to December, 2012, we sought to evaluate anesthesia residents' perspectives, perceptions and experiences within the local environment to understand the issues that affect postoperative pain management.

\section{METHODS}

A qualitative needs assessment was conducted, which included a focus group of anesthesia residents. Three goals were set for these focus group sessions: to assist in the development of a culturally appropriate questionnaire for use among a diverse hospital staff; to help guide the development of an effective knowledge translation strategy in acute pain management; and to identify the means for future evaluation of this knowledge translation strategy.

\section{Participants}

Institutional ethics approval was obtained from both Queen's University (Kingston, Ontario) and the National University of Rwanda, and verbal informed participant consent was obtained from participants. Anesthesia residents were chosen for the focus group sessions because they have insight into the use of pain management strategies, including opioid administration, across a variety of health care disciplines that are involved in pain management, and because of their involvement with the CASIEF program. Because of this, they were uniquely positioned to provide key information about the root causes and conditions of current pain management practices. Residents were also targeted because they have experience (and memory) of training practices and protocols, but also have experience and perspective regarding the day-to-day management of pain within the hospital. Finally, residents constituted a convenient audience to question because their seminars have been previously organized and held weekly. Through CASIEF, a relationship with residents had been established and time away from clinical duties was provided, making this a practical group to study. By contrast, medical and nursing staff were in short supply and could not be assembled with simultaneous time away from clinical duties.

\section{Data collection and analysis}

Data were collected on two consecutive weekly sessions. The focus group methodology was chosen in an attempt to separate individual opinion and personal bias from the broader context of group and institutional norms and culture, which was the focus of the current needs 
analysis $(28,29)$. Three of the authors (AJ, JP, RM) conducted two focus group sessions, each with the same group of 10 anesthesia residents. The sessions were conducted after the residents' usual weekly core teaching seminars at 15:00. There were no time constraints on the length of the focus groups. In the morning, before the start of the seminar, it was announced that focus groups were intended to be conducted at the end of that day. Participants were instructed that the focus groups were completely voluntary, and all verbally consented to participate. An interview manual was constructed based on previous research and understanding, with the intent of directing the discussion toward generalized topics focused on identifying which issues affect pain management. However, the intent (and the eventuality) of the focus group was to explore multiple dimensions of pain control from the perspective of Rwandan anesthesiology residents. As such, the conversation was allowed to diverge and re-emerge around multiple dimensions in an effort to glean a thorough understanding of the residents' lived experiences. On analysis, emergent codes included general background of pain management experience, perceptions of postoperative pain management, impact of pain management on clinical outcomes, and conflicts between theoretical best practices and the realities of practice (including resource availability, allocation, costs, and pain evaluation). Prompting was provided to participants when the discussion lagged or migrated away from the topic of pain management. Audiotaping and transcription were not used due to participant concerns regarding the confidentiality of the topics to be discussed. However, once participants recognized the minimal risks involved, they appeared to be free to divulge their individual thoughts. Responses were recorded anonymously through researcher field notes.

The field notes were analyzed using interpretative (RM and JP) and descriptive (RM, JP and AJ) phenomenological approaches, respectively. As experienced academic anesthesiologists, RM and JP brought a wealth of clinical knowledge to the analysis that was used "as a valuable guide to inquiry" (30). However, both researchers also remained careful not to presuppose cultural understandings. They interpreted comments regarding cultural phenomena using a descriptive phenomenological approach in an effort "to strip away prior experiential knowledge and personal bias so as not to influence the description of phenomenon at hand" (31). Relying on expertise in health policy, AJ used a descriptive approach throughout. Analysis included coding, categorizing and theming notes obtained by each researcher at each focus group. After categorizing, rationalizing and journaling their analysis of individual notes, the researchers compared and contrasted their findings through discussion.

\section{RESULTS}

Participants' perspectives regarding each topic area are presented. Participants' comments were condensed into codes reflective of their lived experiences. Quotations from participants are provided when recorded verbatim to support reported codes; however, paraphrasing may have occurred due to limitations implicit to note taking.

General patient and medical practice management

Lack of communication with patients about pain management: Residents referred to a lack of communication with patients regarding pain management. One participant stated that "patients were rarely informed preoperatively about postoperative pain management options". Others agreed, noting that patients remained uninformed unless a specific procedure (eg, regional block) had been planned. Barriers identified by the participants included patients' level of education, cultural expectations (eg, a paternalistic approach to health care) and time available for discussion.

Lack of continuity of pain management postoperatively: There was agreement that the anesthesiologist is the primary person responsible for prescribing analgesia while in the recovery room, and that surgeons and anesthesia technicians share the responsibility with the anesthesiologists for following patients and adjusting medications in that setting. A participant noted that, once discharged from the recovery room, "it is primarily the surgeons and nurses who are responsible for postoperative pain management, although this is dependent on the type of surgical ward". For example, in the intensive care unit (ICU), nurses tend to be responsible for formally assessing pain; therefore, they tend to be involved more directly with postoperative pain management. Others in the group added that postoperative pain management modalities implemented in the recovery room did not continue after the patient was transferred to the ward. Because "anesthesia providers were rarely involved in changes to the treatment plan on the ward", residents may not fully understand the ongoing process of pain management. This would indicate that nurses underwrote much of the pain management regimens on the wards. This concerned residents, because many noted that postoperative pain management is only provided on the wards when there is a patient complaint, rather than by regular administration or protocols. Residents believed that "patient comfort and satisfaction", postoperative morbidity (ie, fewer complications), length of stay (length of stay was viewed to be more expensive than analgesics), recovery time and costs were the outcomes most important for determining postoperative pain management processes. There was less concordance regarding the importance of mortality, with one participant noting that "postoperative mortality was slightly important" when considering proper postoperative pain management.

\section{Knowledge of postoperative pain management}

'Hands-on' pain management training: Residents described little formal 'hands-on' pain management training. Some participants stated that "no training was received", while others stated that they acquired their knowledge "from books and didactic training". When a participant commented that there is "insufficient training", others in the group emphasized that "there was a disconnect between didactic teaching and clinical practice, despite available didactic training materials". For example, residents were asked about their knowledge of analgesic treatment for postoperative pain management during the first two postoperative days. Based on their training, they responded that "depending on the surgical procedure, the following types of treatment are indicated (although not necessarily provided) on the general surgical ward: continuous epidural analgesia, peripheral blocks and local infiltration, or orally or rectally administered analgesics (including tramadol, anti-inflammatory drugs, opioids and paracetamol)". Others in the group added that "few peripheral blocks are used due to the lack of anesthesiologists' and residents' experience and availability of ultrasound equipment; regional anesthesia is limited predominantly to spinal and intravenous blocks". There was concordance that the "system provides little opportunity to put knowledge into practice". The main barriers to hands-on training listed by participants included a discussion about how the local anesthesiologists and surgeons do not have sufficient time to provide practical training in postoperative pain management, and how there was still dependency on CASIEF physicians and pain nurses for this training. The lack of time available to anesthesiologists and surgeons to provide hands-on training and the limited availability of equipment may represent a need to emphasize resource allocation.

\section{Pain evaluation}

Inconsistent use of pain evaluation tools: The residents believed that protocols to regularly assess postoperative pain on the surgical ward(s) were not being used. One participant stated that "the norm is to only look for body language or verbal cues from patients to assess the level of pain". If body language is used to gauge the level of pain, there is inconsistency and interpersonal subjectivity in the administration of analgesics. When asked whether there should be a threshold level of pain at which rescue analgesia should be routinely given, the majority opinion was that "if the patient does not complain of pain, there is no need to treat with potent analgesics, assuming that the patient is given paracetamol". Without a threshold, even administration of paracetemol becomes varied across medical care practitioners, as well as among patients. When asked whether postoperative pain should routinely be measured using pain scores at rest and during movement, there was 
TABLE 1

Themes and subthemes arising from the focus groups

\begin{tabular}{l}
\hline General patient and medical practice management \\
\hline Lack of communication with patients about pain management \\
Patients' level of education \\
Cultural expectations \\
Time available for discussion \\
Lack of continuity of pain management from recovery room to wards \\
Communication between anesthesiologists and surgeons/nurses \\
Pain management only provided upon patient complaint \\
Different emphases on important factors for pain management \\
Knowledge of postoperative pain management \\
\hline Insufficient hands-on pain management training \\
Emphasis on books and didactic training \\
Disconnect between didactic teaching and clinical practice \\
Few opportunities at system level to put knowledge into practice \\
Insufficient time by anesthesiologists to provide hands-on training \\
Limited availability of equipment \\
Pain evaluation \\
\hline Inconsistent use of pain evaluation tools \\
Subjective perceptions of pain \\
Inexistent perception of pain threshold \\
Incosistent training of pain assessment tools \\
Lack of time and resources for training \\
Protocol management: Institutional/system issues \\
Lack of awareness of existing protocols \\
Protocols not displayed on walls \\
No delineation of responsibilities \\
Insufficient drugs, equipment, personnel \\
Lack of experience
\end{tabular}

unanimous agreement for "measuring it at rest". There was uncertainty regarding the need to measure pain during movement out of concern for overestimating the pain experienced. A participant noted that a visual analogue scale for pain assessment is available to be used in the ICU; however, only ICU nurses are trained to do so. Others in the group added that "these assessments are often not conducted due to lack of resources" (personnel and time). Exclusive formal use of pain assessment in the ICU perpetuates differences in pain administration across sectors within the hospital. Limited resources of drugs and personnel appear to be a resounding issue. Furthermore, participants noted that "when postoperative pain scores are measured, they are documented on the patient's chart". There was less concordance regarding whether "these assessments are used to alter treatment", with one participant noting that "pain is rarely evaluated on the surgical wards". Fundamentally, a widespread use of pain assessment scales and thresholds is only likely to become pervasive if a cultural paradigm shift is made from beliefs regarding body language and verbal cues used as markers for pain assessment to other, more formal, types of pain assessment. The addition of chart records would improve communication among health care workers with respect to the patients, as well as ensure continuity of care within patients and across settings/wards. Operational feasibility is also crucial for there to be sufficient resources for pain assessment in all sectors across all health care personnel because lack of resources appears to represent an operational barrier.

Institutional/system issues related to protocol management Lack of awareness of existing protocols: Residents believed that although some pain management protocols have been developed, "they are not regularly employed". Others indicated that "they should be displayed on walls in all units (ICU, recovery room, wards) and clearly indicate the responsibilities of the different groups of personnel: nurses, technicians, anesthesiologists and surgeons". There was widespread agreement that protocols could address the coordination of patients' care in terms of pain management, and one participant noted that "these protocols should be reviewed by many health care sectors, for example, the nursing care team, administration and, ultimately, by anesthesiologists". Some of the participants appeared to be aware of the existence of protocols, as well as recognizing the importance of coordination of care. Others in the group added that this would "ensure successful implementation". Team work could be fostered if residents were to participate in the implementation of protocols. In this area, participants appeared to be already entrenched in a culture of cooperation towards a single goal: optimal patient care.

\section{Resource allocation}

Lack of resources available for pain management: Residents noted a general lack of resources available for pain management. General lack of resources listed by participants included "drugs, equipment and personnel". They universally remarked that "this limitation of resources constitutes a barrier to implementing postoperative pain management". One participant noted that "although this perception extends to other important hospital services, resources specific to pain management including ultrasound and epidural equipment, and drugs with limited availability (eg, ketamine)", there was also the perception of a lack of experience in using these resources. When asked more generally what anesthesia resources they would add if additional funding were available, there was agreement that they would "add space, new construction, ventilators, specialized equipment (eg, ultrasound), monitoring for anesthetic machines (eg, capnography), laboratories, examination capabilities and technicians". It is important to note that the pervasive awareness of the surroundings and limitations of resources therein represents insightfulness and a good footing for the impetus for these residents (and eventually staff members) to take part in planning and resource allocation. This involved planning process would be made possible as long as local health care professionals are able to have a voice in the resource allocation process within Rwanda's health care system. At the very least, they need to have a voice in how resource allocation pertains to pain management.

Table 1 summarizes the themes and subthemes identified. The residents agreed that, based on these five themes, it will be possible to develop a questionnaire that would represent hospital personnel's frame of reference, taking into account their diverse backgrounds, education and expertise.

\section{DISCUSSION}

The present study captured anesthesia residents' perspectives, perceptions and experience with respect to pain management in a large teaching hospital in Kigali, Rwanda. An important gap in knowledge translation (communication among stakeholders and with patients; on-the-job training; use of protocols; widespread pain assessment; participation in resource allocation decisions) has been confirmed and described. The present work revealed that, although the didactic portion of the residency training program was perceived to be strong and comprehensive, residents believed that there was little formal pain management training, especially with respect to hands-on practice. Furthermore, even though training has been provided by CASIEF, demonstrated by residents' acknowledgement of their completeness of didactic training, there was an unanimous wish for hands-on experience with respect to structured training. A future survey focusing on general patient and medical practice management will serve to elucidate how hands-on experience can be improved.

In the residents' perception, it was discovered that there is little communication between medical staff and patients regarding pain management options, there is no regular assessment of pain, and administration of pain modalities is based mainly on patient distress. Thus, pain management relied primarily on interpreting body language and verbal cues from patients, rather than on protocols for formal assessment and treatment. This highlights a long-standing challenge and 
generally results in suboptimal pain management (16). Formal pain evaluation was only performed according to protocol in the ICU, and residents suggested the posting of protocols to encourage more consistent practice outside of that area. They expressed concern that pain management is a low priority, particularly postoperatively on the ward, which leads to under-use of limited available resources and decreases the potential for realistic interventions to advance pain management at minimal additional cost. As Cordts et al (32) found in their assessment of pain management for hospitalized orthopedic patients, there is a pervasive inadequacy in on-the-job learning that is widespread. CASIEFs future role may be to increase focus on hands-on training for the residents.

As stated above, the influence of adequate postoperative pain management was viewed to be important in a multitude of areas (patient comfort and satisfaction, postoperative morbidity, length of stay, recovery time and costs). The influence of proper postoperative management regarding mortality was deemed to be less important. The diminished relative emphasis regarding mortality may reflect actual or cultural experiences. This is similar to what is experienced elsewhere in the world (33). Continued emphasis on ensuring that basic needs are met by providing accurate pain assessment and prompt treatment would shift the focus away from mortality, toward sustaining life. The role of pain management can be viewed through the lens of health-seeking behaviour and cultural norms from a sociomedical standpoint (accessibility, costs, acceptability), or from an anthropological viewpoint (focusing primarily on etiological concepts). When these two approaches (epidemiological and anthropological) can be used in conjunction, there may be an improvement in adequately and environmentally adapted pain management, especially given Rwanda's unique historical and cultural experiences $(34,35)$.

Future planning for sustainability and autonomy in perioperative practice should include improvement in training, education of all health care providers and adequate provision of resources. An emphasis on the establishment of multidisciplinary teams, including surgeons, anesthesiologists and nurses, appears to be a prerequisite for improvement in pain management, as found by Bardiau et al (36) in their assessment of an intervention to enhance postoperative pain management. They conducted a study involving the implementation of an acute pain service in a 1000-bed hospital and found that a significant improvement in pain relief resulted. There are many unfulfilled needs within pain management, which may be the result of inadequate prioritization and a lack of availability of resources. Because pain assessment protocols are incorporated in the ICU in Kigali, residents believed that appropriate pain assessment may be implemented on other units. With the development of a future survey of the diverse members of the pain management team, it will be possible to determine how best to make such a protocol part of the daily patient management practice.

\section{REFERENCES}

1. World Health Organization. Questions and answers on health and human rights. In: Health and Human Rights Publication Series World Health Organization. July, 2002. Issue 1.

2. Brennan F, Cousins MJ, Carr DB. Pain relief as a human right. Pain 2004;112:1-4.

3. International Association for the Study of Pain (IASP). Declaration of Montreal. <www.iasp-pain.org/DeclarationofMontreal?navItemN umber=582 $>$ (Accessed March 29, 2015).

4. Suleiman A, Burton AW. Introducing interventional pain services in a large African teaching hospital: Challenges and opportunities. Pain Pract 2013;13:663-6.

5. Hosseinpoor AR, Bergen N, Mendis S, et al. Socioeconomic inequality in the prevalence of noncommunicable diseases in lowand middle-income countries: Results from the World Health Survey. BMC Public Health 2012;12:474.

6. Elzahaf RA, Tashani OA, Unsworth BA, Johnson MI. The prevalence of chronic pain with an analysis of countries with a human development index less than 0.9: A systematic review with meta-analysis. Curr Med Res Opin 2012;28:1221-9.
The present study is the first to report on perceptions about postoperative pain management in Kigali, Rwanda. Limitations of the present study include a limited group of personnel (anesthesia trainees) and a focused area of questioning (postoperative pain management, as opposed to other services). In turn, the present work will contribute to the development of culturally appropriate and relevant questions to be addressed in a further study involving residents, staff anesthesiologists, surgeons and nurses, before the implementation of a pain management strategy. Future studies will address the five general areas identified: general patient and medical practice management; knowledge base regarding postoperative pain management; pain evaluation; institutional/system issues related to protocol management; and perceptions about resource allocation and other barriers to implementation.

Further research is needed to assess generalizability to other hospitals in Rwanda (eg, local community facilities) and to other developing countries, when identifying needs and barriers to care among medical and nursing personnel in pain management. Through research, training and knowledge translation among all stakeholders, clinical practice changes and capacity building will help achieve the ultimate goal of ameliorating quality of health care service provided to patients.

In summary, a focus group setting using anesthesia residents was employed to determine opportunities and barriers to postoperative pain management in Kigali, Rwanda. The five general areas (general patient and medical practice management, knowledge base regarding postoperative pain management, pain evaluation, institutional/system issues related to protocol management, and perceptions about resource allocation and other barriers to implementation) uncovered in the present study will form the framework for future questionnaire development directed toward measuring the impact of pain education programming and for creating interdisciplinary practice improvement in Rwanda, which takes into account multiple cultural backgrounds and diverse hospital staff. This information, in addition to assisting in the development of a questionnaire to further assess needs related to pain management education, will be used to promulgate communication among all stakeholders to improve pain management through an integrated education and implementation strategy.

ACKNOWLEDGEMENTS: The authors are grateful to the Canadian Anesthesiologists' Society International Education Foundation for the opportunity to teach and learn from colleagues in Rwanda. The authors also thank colleagues in Rwanda for their hospitality, and willingness to work together to improve pain management among their patients. Drs Gaston Nyirigira, Rosemary Wilson and Elizabeth VanDenKerkhof are thanked for their input into this project.

DISCLOSURES: The authors certify that he or she, or a member of his or her immediate family has no commercial association (ie, consultancies, stock ownership, equity interest, patent/licensing arrangements) that maypose a conflict of interest in connection with the published article.

7. Bond M. Pain education in developing countries and responses to them by the International Association for the Study of Pain. Pain Res Manag 2011;16:404-6.

8. Macpherson C, Aarons D. Overcoming barriers to pain relief in the Caribbean. Dev World Bioeth 2009;9:99-104.

9. Mathers CD, Lopez AD, Murray CJL. The burden of disease and mortality by condition: Data, methods, and results for 2001. In: Lopez AD, Mathers CD, Ezzati M, et al, eds. Global Burden of Disease and Risk Factors, 1st edn. New York: The World Bank and Oxford University Press, 2006:45-180.

10. Australian and New Zealand College of Anaesthetists and Faculty of Pain Medicine. Acute Pain Management: Scientific Evidence. 2nd ed, 2005:14-16.

11. VanDenKerkhof EG, Hopman WM, Goldstein DH, et al. Impact of perioperative pain intensity, pain qualities, and opioid use on chronic pain after surgery: A prospective cohort study. Reg Anesth Pain Med 2012;37:19-27. 
12. Gerbershagen HJ, Ozgur E, Dagtekin O, et al. Preoperative pain as a risk factor for chronic post-surgical pain - six month follow-up after radical prostatectomy. Eur J Pain 2009;13:1054-61.

13. Aisuodionoe-Shadrach OI, Olapade-Olaopa EO, Soyannwo OA. Preoperative analgesia in emergency surgical care in Ibadan. Cent Afr J Med 2001;1:70-4.

14. Faponle AF, Soyannwo OA, Ajayi IO. Postoperative pain therapy: A survey of prescribing patterns and adequacy of analgesia in Ibadan, Nigeria. Cent Afr J Med 2001;47:70-4.

15. Size M, Soyannwo OA, Justins DM. Pain management in developing countries. Anaesthesia 2007;62:38-43.

16. Hodges SC, Mijumbi C, Okello M, McCormick BA, Walker IA, Wilson IH. Anesthesia services in developing countries: Defining the problems. Anaesthesia 2007;62:4-11.

17. Coulling S. Nurses' and doctors' knowledge of pain after surgery. Nurs Stand 2005;19:41-9.

18. Agency for Health Care Policy and Research. Clinical practice guideline cancer pain management. Rockville, MD: US Department of Health and Human Services, 1994.

19. Davis MP, Walsh D. Epidemiology of cancer pain and factors influencing poor pain control. Am J Hosp Palliat Care 2004;21:142.

20. Sun VC, Borneman T, Ferrell B, Piper B, Koczywas M, Choi K. Overcoming barriers to cancer pain management: An institutional change model. J Pain Symptom Manag 2007;34:359-69.

21. Turk DC. Customizing treatment for chronic pain patients: Who, what and why? Clin J Pain 1990;6:255-70.

22. Glajchen M. Chronic pain: Treatment barriers and strategies for clinical practice. J Am Board Fam Pract 2001;14:211-8.

23. Twagirumugabe T, Carli F. Rwandan anesthesia residency program: A model of north-south educational partnership. Int Anesthesiol Clin 2010;48:71-8.
24. Shrestha BM, Rana NB. Training and developing anesthesia in Nepal-1985 to 2005. Can J Anesth 2006;53:339-43.

25. Farmer PE, Kim JY. Surgery and Global Health: A view from beyond the OR. World J Surg 2008;32:533-6.

26. Omahen DA. The 10,000-hour rule and residency training. CMAJ 2009;180:1272.

27. Petroze RT, Nzayisenga A, Rusanganwa V, Ntakiyiruta G, Calland JF. Comprehensive national analysis of emergency and essential surgical capacity in Rwanda. Br J Surg 2012;99:436-43.

28. Morgan DL. The Focus Group Guide Book. London: Sage Publications. 1998.

29. Gill P, Stewart K, Tresure E, Chadwick B. Methods of data collection in qualitative research: Interviews and focus groups. Brit Dent J 2008;204:291-5.

30. Lopez KA, Willis DG. Descriptive versus interpretive phenomenology: Their contributions to nursing knowledge. Qual Health Res 2004;14:726-35.

31. Wojnar DM, Swanson KM. Phenomenology: An exploration. J Holistic Nurs 2007:25;172-80.

32. Cordts GA, Grant MS, Brandt LE, Mears SC. Assessment of pain management for hospitalized orthopedic patients. Orthopedics 2011;34:368-73.

33. Taylor A, Stanbury L. A review of postoperative pain management and the challenges. Trends Anaesth Crit Care 2009;20:188-94.

34. Kroeger A. Anthropological and socio-medical health care research in developing countries. Soc Sci Med 1983;17:147-61.

35. Buckley-Zistel S. Remembering to forget: Chosen amnesia as a strategy for local coexistence in post-genocide Rwanda. Africa 2006;76:131-51.

36. Bardiau F, Taviaux NF, Albert A, Boogaerts JG, Stadler M. An intervention study to enhance postoperative pain management. Anesth Analg 2003;96:179-85. 


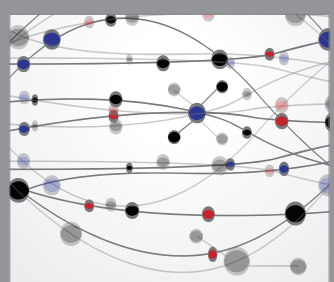

The Scientific World Journal
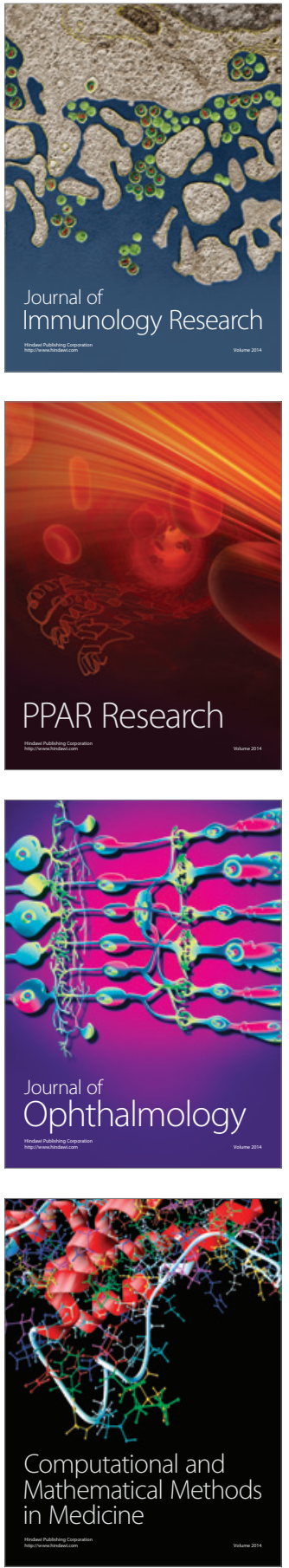

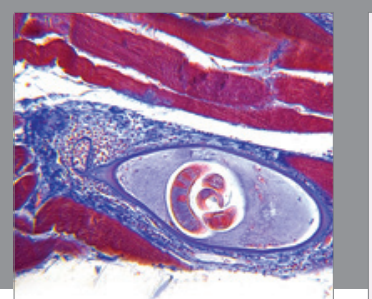

Gastroenterology Research and Practice

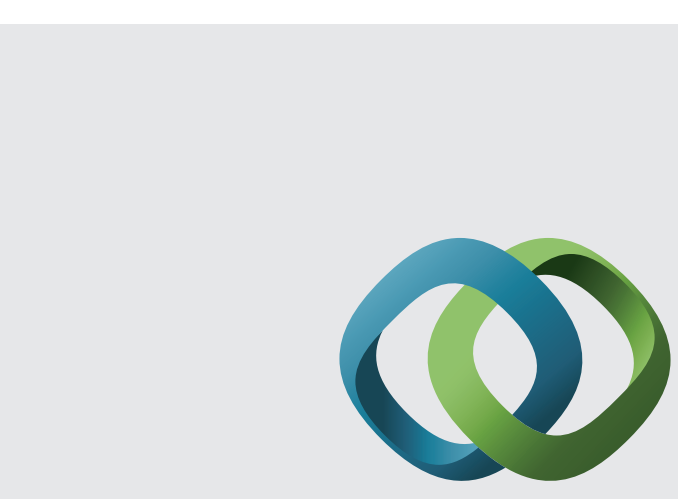

\section{Hindawi}

Submit your manuscripts at

http://www.hindawi.com
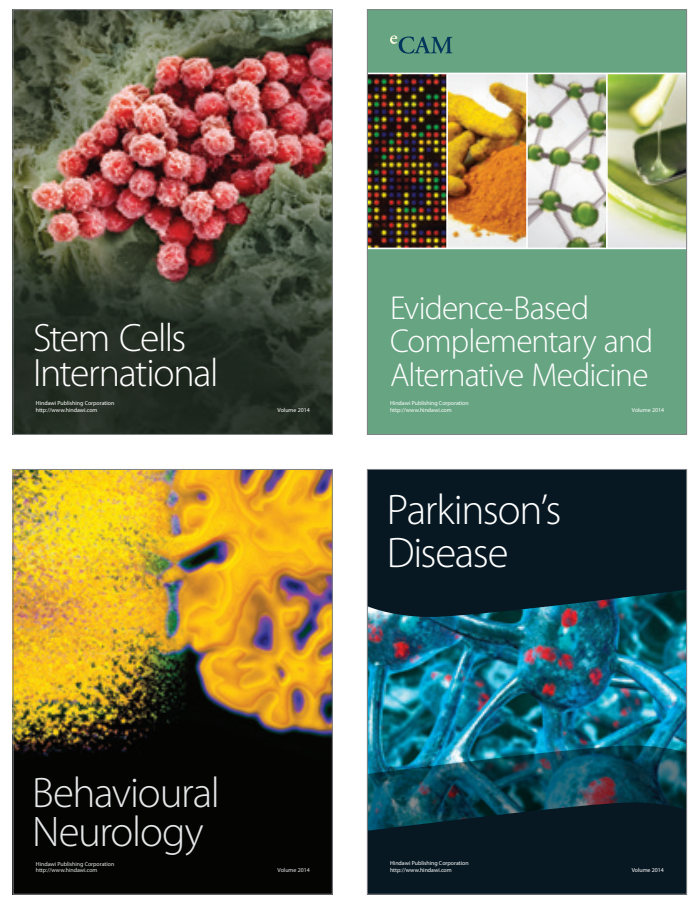
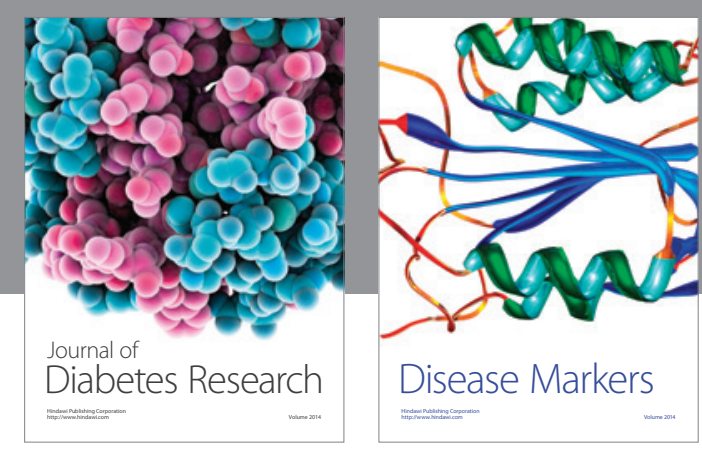

Disease Markers
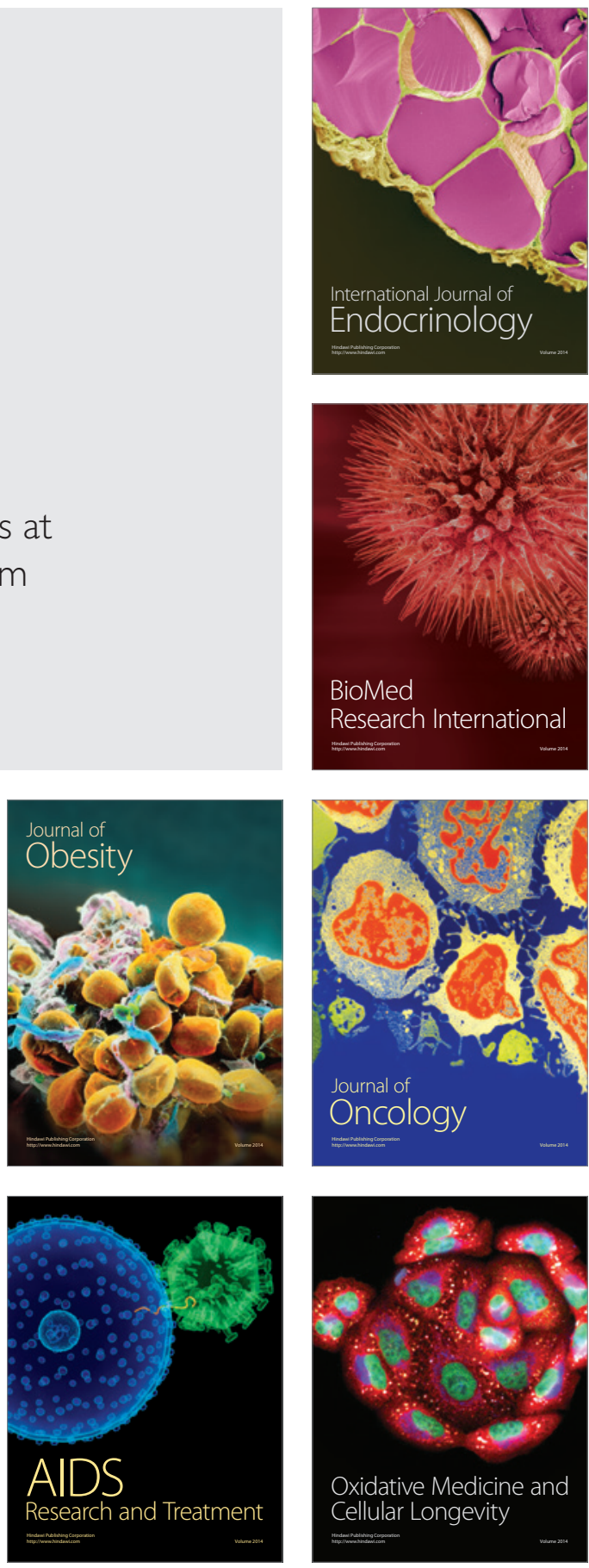